\title{
Neuroprotective mechanism involved in spinal cord stimulation postconditioning
}

\author{
Huixian Li, MD, ${ }^{a}$ Xiuhua Dong, MM, ${ }^{\mathrm{b}}$ Weiping Cheng, MM, ${ }^{\mathrm{b}} \mathrm{Mu}$ Jin, $\mathrm{MD},{ }^{\mathrm{c}}$ and Deqiang Zheng, $\mathrm{PhD}^{\mathrm{d}}$
}

\section{ABSTRACT}

Objectives: Delayed paraplegia developed postoperatively after thoracoabdominal aneurysm surgery is primarily associated with spinal cord ischemia/reperfusion injury. Our previous study suggested that spinal cord stimulation postconditioning protected the spinal cord from ischemia/reperfusion injury through microglia inhibition. In this study, we further investigated whether $\alpha 7$ nicotinic acetylcholine receptors were involved in the neuroprotective mechanism of spinal cord stimulation.

Methods: Rabbits were randomly assigned to sham, control, $2 \mathrm{~Hz}, \alpha$-bungarotoxin, and $2 \mathrm{~Hz}$ - $\alpha$-bungarotoxin groups ( $\mathrm{n}=24 /$ group). Transient spinal cord ischemia was performed on all rabbits except rabbits in the sham group. Rabbits in the control group received no further intervention, rabbits in the $2 \mathrm{~Hz}$ group were given $2 \mathrm{~Hz}$ spinal cord stimulation, rabbits in the $\alpha$-bungarotoxin group received prescribed intrathecal $\alpha$-bungarotoxin ( $\alpha$-bungarotoxin, a specific $\alpha 7$ nicotinic acetylcholine receptor antagonist) injections, and rabbits in the 2 $\mathrm{Hz}$ - $\alpha$-bungarotoxin group received both $\alpha$-bungarotoxin injections and $2 \mathrm{~Hz}$ spinal cord stimulation. Hind-limb neurologic function was assessed, and spinal cord histologic examination, terminal deoxynucleotidyl transferase-mediated dUTP nick end labeling staining, and microglia staining were performed at 8 hours, 1 day, 3 days, and 7 days of reperfusion.

Results: Rabbits in the $2 \mathrm{~Hz}$ group had significantly better neurologic functions, more $\alpha$-motor neurons, and lower terminal deoxynucleotidyl transferasemediated dUTP nick end labeling-positive neuron rates and microglia area/anterior horn area ratios (microglia area ratios) than the control group. The neurologic functions of the $\alpha$-bungarotoxin group were significantly worse than those of the control group, whereas other results were not significantly different from the control group. The results of the $2 \mathrm{~Hz}-\alpha$-bungarotoxin group were insignificant to the control group except for the terminal deoxynucleotidyl transferase-mediated dUTP nick end labeling-positive neuron rates, which were significantly lower than in the control group.

Conclusions: The neuroprotective effects of spinal cord stimulation postconditioning against spinal cord ischemia/reperfusion injury were partially mediated by activating $\alpha 7$ nicotinic acetylcholine receptors. (J Thorac Cardiovasc Surg 2020;159:813-24)

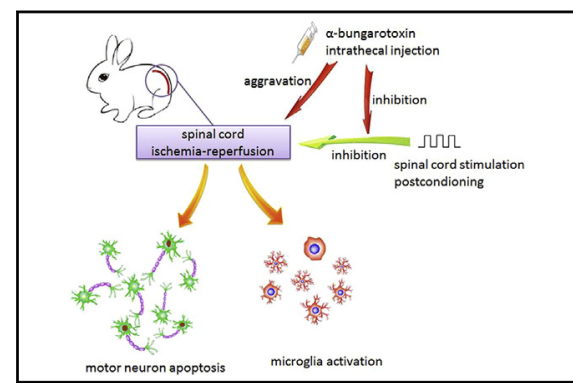

Neuroprotective mechanism involved in SCS postconditioning.

Central Message

The neuroprotective effects of $2 \mathrm{~Hz}$ SCS postconditioning in spinal cord $\mathrm{I} / \mathrm{R}$ injury are partially mediated by activation of $\alpha 7 \mathrm{nAchR}$.

\section{Perspective}

Delayed paraplegia due to spinal cord I/R injury remains one of the most severe complications of thoracoabdominal aneurysm surgery. Our study demonstrated that $2 \mathrm{~Hz}$ SCS postconditioning could protect the spinal cord against I/ $\mathrm{R}$ injury in rabbits. The mechanism is related to activation of $\alpha 7 \mathrm{nAchR}$

See Commentaries on pages 825 and 827.

\footnotetext{
From the ${ }^{\mathrm{a}}$ Department of Cardiac Surgery, The First Hospital of Tsinghua University, Beijing, China; ${ }^{b}$ Department of Anesthesiology, Beijing Anzhen Hospital, Beijing Institute of Heart, Lung and Blood Vessel Diseases, Capital Medical University, Beijing, China; ' Department of Anesthesiology, Beijing Friendship Hospital, Capital Medical University, Beijing, China; and ${ }^{\mathrm{d}}$ Department of Epidemiology and Health Statistics, School of Public Health, Capital Medical University, Beijing, China.

This research was funded by Beijing Natural Science Foundation (No. 7152046). The funder had no role in the study design, data collection and analysis, decision to publish, or preparation of the manuscript.

Received for publication June 3, 2018; revisions received Feb 25, 2019; accepted for publication March 13, 2019; available ahead of print April 25, 2019.
}

\footnotetext{
Address for reprints: Weiping Cheng, MM, Department of Anesthesiology, Beijing Anzhen Hospital, Capital Medical University, Beijing Institute of Heart, Lung and Blood Vessel Diseases, Beijing, China; Mu Jin, MD, Department of Anesthesiology, Beijing Friendship Hospital, Capital Medical University, Beijing, China and Deqiang Zheng, PhD, Department of Epidemiology and Health Statistics, School of Public Health, Capital Medical University, Beijing, China (E-mail: ch_eng9735@sina.com or jinmu0119@hotmail.com or dqzheng@ccmu.edu.cn). $0022-5223 / \$ 36.00$

Copyright (C) 2019 Published by Elsevier Inc. on behalf of The American Association for Thoracic Surgery

https://doi.org/10.1016/j.jtcvs.2019.03.048
} 


\section{Abbreviations and Acronyms \\ $\alpha$-BGT $=\alpha$-bungarotoxin \\ $\alpha 7 \mathrm{nAchR}=\alpha 7$ nicotinic acetylcholine receptor \\ $\mathrm{CI}=$ confidence interval \\ $\mathrm{HE} \quad=$ hematoxylin and eosin \\ Iba1 = ionized calcium binding adaptor molecules 1 \\ $\mathrm{I} / \mathrm{R}=$ ischemia/reperfusion \\ SCS $=$ spinal cord stimulation \\ TUNEL $=$ terminal deoxynucleotidyl transferase- mediated dUTP nick end labeling}

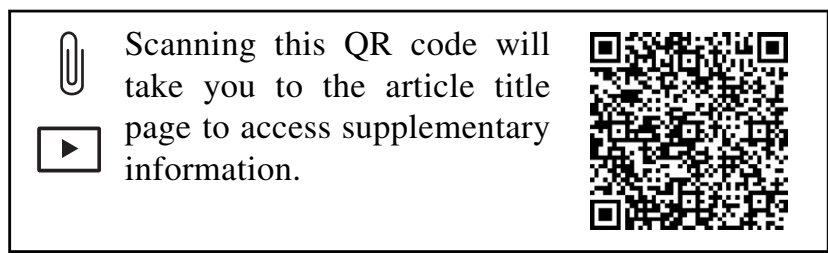

Delayed paraplegia is one of the most unpredictable and severe complications of thoracoabdominal aneurysm surgery. ${ }^{1}$ Although the pathogenesis of delayed paraplegia is still not fully understood, the root of it is believed to be spinal cord ischemia/reperfusion (I/R) injury. Strategies to protect the spinal cord against I/R injury have been extensively studied. Our previous study suggested that spinal cord stimulation (SCS) postconditioning could protect the spinal cord from I/R injury, ${ }^{2}$ and the mechanism was related to microglia inhibition. ${ }^{3}$ As resident immune cells of central nervous system, microglia are activated by spinal cord $\mathrm{I} / \mathrm{R}$ and strongly express inflammatory cytokines, such as tumor necrosis factor- $\alpha$, interleukin- $1 \beta$, and interleukin- 6 , which evoke robust inflammation and eventually lead to neuron apoptosis and death, ${ }^{4-7}$ whereas microglia inhibition ${ }^{8-10}$ demonstrates neuroprotective effects. For example, studies by Smith and colleagues ${ }^{9}$ and Takeda and colleagues ${ }^{10}$ on mice and rat models of spinal cord I/R injury demonstrated that microglia inhibition significantly preserved animals' hind-limb neurologic function and spinal cord motor neurons, respectively. As for the mechanism of microglia inhibition, previous studies of cerebral ischemia indicated that pharmaceutical activation of $\alpha 7$ nicotinic acetylcholine receptors ( $\alpha 7 \mathrm{nAchRs})$ expressed on microglia by the administration of synthetic $\alpha 7 \mathrm{nAchR}$ agonists $^{11,12}$ or nicotine ${ }^{13}$ could inhibit microglia activation, attenuate inflammatory cytokine production, and significantly decrease cerebral infarction volume and improve neurologic function. In addition, right vagus nerve stimulation also exerted neuroprotective effects through $\alpha 7 \mathrm{nAchR}$-mediated microglia inhibition in brain $\mathrm{I} / \mathrm{R}$ injury. ${ }^{14,15}$ Taken together, these studies suggest $\alpha 7 \mathrm{nAchR}$ as a regulator of microglia activation and a neuroprotective target. It is unknown whether $\alpha 7 \mathrm{nAchRs}$ are involved in the neuroprotective effects of $2 \mathrm{~Hz}$ SCS postconditioning. By using intrathecal injection of $\alpha$-bungarotoxin ( $\alpha$-BGT) (a subtype-selective irreversible $\alpha 7 \mathrm{nAchR}$ antagonist), the current study investigated the role of $\alpha 7 \mathrm{nAchRs}$ in the neuroprotective effects of $2 \mathrm{~Hz}$ SCS postconditioning against spinal cord I/R injury.

\section{MATERIALS AND METHODS}

Experimental protocols were approved by the animal care and use committee of the Capital Medical University. Male New Zealand white rabbits weighing 2.5 to $3.0 \mathrm{~kg}$ were used in this study. All rabbits received humane care in compliance with the "Guide for the Care and Use of Laboratory Animals" established by National Institutes of Health Publication No. 85-23, revised in 1996, and in compliance with ARRIVE guidelines.

\section{Implantation of Stimulation Electrode and PE-10 Tube}

Implantation of an epidural stimulation electrode was performed as previously reported. ${ }^{2}$ Briefly, the rabbit was anesthetized intravenously with pentobarbital $30 \mathrm{mg} / \mathrm{kg}$ through the marginal ear vein and kept spontaneously breathing, and then placed in a prone position. A 2- to 3-cm midline incision was made at the L3-5 level. The vertebral muscle of the L3-4 interval was bluntly dissected to expose the intervertebral ligament. After the upper half of the spinouts process of L4 was removed, the intervertebral ligament was cut open longitudinally. A stimulation electrode fabricated in the laboratory was introduced through the incision into the epidural space $1.5 \mathrm{~cm}$ caudally. The electrode was sutured to the muscles and routed through the skin. The incision was then closed layer by layer.

Subarachnoid PE-10 tube implantation was used for injection of $\alpha$-BGT. The implantation method was as follows. After implantation of the epidural stimulation electrode, another 2- to 3-cm midline incision was made at the L7-S1 level. The vertebral muscle of the L7-S1 interval was bluntly dissected to expose the intervertebral ligament. After puncturing the ligament with a spinal needle, a segment of PE-10 tube was introduced through the needle hole $3 \mathrm{~cm}$ cephalic into the subarachnoid space. The desired position of the PE-10 tube was confirmed by smooth aspiration of cerebrospinal fluid with a syringe and confirmed again when harvesting the spinal cord. The PE-10 tube was sutured to the muscles and routed through the skin. The incision was then closed layer by layer. Rabbits were allowed to recover for 3 days, and those showing no sign of neurologic defect entered the next stage of the experiment.

\section{Induction of Spinal Cord Ischemia}

Spinal cord ischemia was induced by intraluminal balloon occlusion of the infrarenal abdominal aorta as previously reported, ${ }^{2}$ which is shown in Video 1. Briefly, rabbits were anesthetized with intravenous injection of pentobarbital $30 \mathrm{mg} / \mathrm{kg}$ and placed in the supine position. Both femoral arteries were exposed. A 22-gauge venous catheter was inserted into the left femoral artery to monitor distal arterial blood pressure. A 2F Fogarty arterial embolectomy catheter (Edwards Lifesciences, Irvine, Calif) was advanced $15 \mathrm{~cm}$ retrograde to the abdominal aorta via the right femoral artery, with the tip of the catheter 0.5 to $1.0 \mathrm{~cm}$ beneath the left renal artery (the inserting length of the catheter was confirmed by preliminary experiment). Spinal cord ischemia was induced by inflating the catheter balloon with 0.15 to $0.2 \mathrm{~mL}$ air to occlude the infrarenal abdominal aorta. Successful distal aorta occlusion was confirmed by an immediate and sustained decrease of distal artery blood pressure to approximately $10 \mathrm{~mm} \mathrm{Hg}$. After 28 minutes of occlusion, the balloon catheter was deflated. Both catheters 


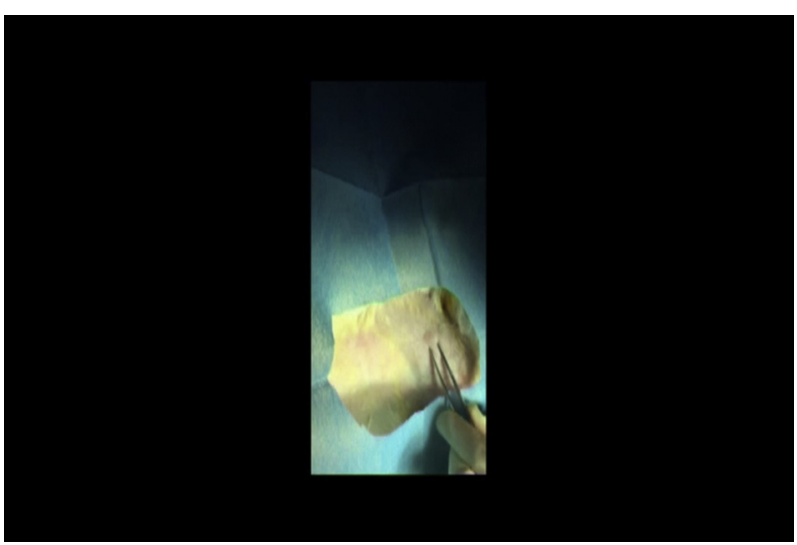

VIDEO 1. Video of spinal cord I/R procedure. The anesthetized rabbit was put in the supine position. Femoral arteries of both sides were exposed. A $22 \mathrm{G}$ catheter was inserted into the left femoral artery to monitor hind-limb blood pressure. Through the right femoral artery, a 2F Fogarty arterial embolectomy catheter (Edwards Lifesciences, Irvine, Calif) was advanced $15 \mathrm{~cm}$ retrograde to the descending aorta. Spinal cord I/R injury was induced by inflating the catheter balloon with 0.15 to $0.2 \mathrm{~mL}$ air to occlude the infrarenal descending aorta for 28 minutes and then deflating the catheter balloon to regain blood supply of the distal descending aorta. The incisions were closed layer by layer. Video available at: https://www.jtcvs. org/article/S0022-5223(19)30722-6/fulltext.

were removed from the femoral arteries, and the incisions were closed. During the procedure, rectal temperature was monitored and maintained at $38^{\circ} \mathrm{C}$ with an infrared heat lamp and a heating pad.

\section{Experimental Protocol}

Rabbits were randomly assigned to sham, control, $2 \mathrm{~Hz}, \alpha$-BGT, and 2 Hz- $\alpha$-BGT groups ( $n=24$ /group), as shown in Figure 1 . The spinal cord ischemia procedure was performed on all rabbits except those in the sham group, which underwent intra-aortic balloon catheter insertion without aorta occlusion. Rabbits in the control group received no further intervention. Rabbits in the $2 \mathrm{~Hz}$ group received $2 \mathrm{~Hz}$ monopolar SCS (square wave, pulse width $0.2 \mathrm{msec}$, current intensity $90 \%$ motor threshold) at the onset of reperfusion and then daily for 30 minutes. Rabbits in the $\alpha$-BGT group received intrathecal injection of $0.5 \mu \mathrm{g} / \mathrm{kg} \alpha$-BGT (concentration $10 \mu \mathrm{g} / \mathrm{mL}$ ) before the onset of reperfusion and then daily. Rabbits in the $2 \mathrm{~Hz}-\alpha$-BGT group received both subarachnoid injection of $0.5 \mu \mathrm{g} / \mathrm{kg} \alpha$-BGT before the onset of reperfusion and then daily and $2 \mathrm{~Hz} \mathrm{SCS}$ for 30 minutes at the onset of reperfusion and then daily (30 minutes after $\alpha$-BGT injection). Hind-limb neurologic function of rabbits was assessed by using the Jacob scale ${ }^{16}$ at 8 hours, 1 day, 3 days, and 7 days of reperfusion, after which 6 rabbits per group were euthanized by open chest transcardial perfusion of heparinized ice-cold $0.9 \%$ saline under anesthesia. The lumbar enlargement was harvested immediately, fixed in $4 \%$ paraformaldehyde, and embedded in paraffin. Serial $5 \mu \mathrm{m}$-thick transverse sections were cut for histologic examination, terminal deoxynucleotidyl transferase-mediated dUTP nick end labeling (TUNEL) staining, and microglia staining. The number of viable $\alpha$-motor neurons in the ventral horns was counted. The rate of TUNEL-positive motor neurons and the ratio of microglia area/anterior horn area (microglia area ratio) were calculated.

\section{Assessment of Neurologic Function}

Hind-limb neurologic function was assessed by 2 trained observers blinded to grouping at 8 hours, 1 day, 3 days, and 7 days after spinal cord ischemia using the Jacob scale, which ranges from 0 to 5: $0=$ complete paralysis, no detectable movement; $1=$ severe paresis, poor initiative lower-extremity movement, cannot resist gravity, unable to draw legs under body; 2 = good lower-extremity motor function, can resist gravity, able to draw legs under body but cannot hop; $3=$ can hop but not normally, ataxia; $4=$ can hop but not enough flexibility, mild ataxia; $5=$ normal hind-limb locomotion.

\section{Histologic Examination}

Paraffin-embedded spinal cord sections were stained with hematoxylin and eosin (HE) for histologic examination. Viable $\alpha$-motor neurons in the anterior horn were counted by an investigator unaware of group

\begin{tabular}{|c|c|c|c|c|c|c|c|}
\hline sham group & $\mathrm{D} 1$ & $\overline{\mathrm{D} 2}$ & D3 & $\overline{\mathrm{D} 4}$ & $\mathrm{D} 5$ & $\mathrm{D} 6$ & D7 \\
\hline
\end{tabular}

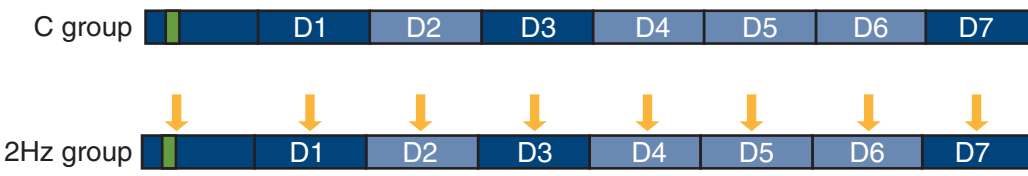

\begin{tabular}{|c|c|c|c|c|c|c|c|}
\hline \multirow[t]{3}{*}{$\alpha$-BGT group } & D1 & $\mathrm{D} 2$ & D3 & $\mathrm{D} 4$ & D5 & D6 & D7 \\
\hline & $\uparrow$ & $\uparrow$ & $\uparrow$ & $\uparrow$ & $\uparrow$ & $\uparrow$ & $\uparrow$ \\
\hline & $\downarrow$ & $\downarrow$ & $\downarrow$ & $\downarrow$ & $\downarrow$ & $\downarrow$ & $\downarrow$ \\
\hline $2 \mathrm{~Hz}-\alpha-\mathrm{BGT}$ group & D1 & $\mathrm{D} 2$ & D3 & D4 & D5 & D6 & D7 \\
\hline
\end{tabular}

FIGURE 1. Grouping and experimental protocol. A total of 120 rabbits were randomly assigned to sham, control, $2 \mathrm{~Hz}, \alpha$-BGT, and $2 \mathrm{~Hz}-\alpha$-BGT groups ( $n=24$ /group). Spinal cord I/R was induced by infrarenal aorta balloon occlusion for 28 minutes on all rabbits except those in the sham group. Rabbits in the control group received no further intervention; rabbits in the $2 \mathrm{~Hz}$ group received 30 minutes $2 \mathrm{~Hz}$ SCS at reperfusion and then daily; rabbits in the $\alpha$-BGT group received intrathecal injection of $\alpha$-BGT $0.5 \mu \mathrm{g} / \mathrm{kg}$ before reperfusion and then daily; rabbits in the $2 \mathrm{~Hz}-\alpha$-BGT group received both $\alpha$-BGT injections and $2 \mathrm{~Hz} \mathrm{SCS}$ at the corresponding time. Rabbits $(\mathrm{n}=6)$ were killed in each group at 8 hours, 1 day, 3 days, and 7 days of reperfusion, respectively. Red mark, surgical procedure without infrarenal aorta occlusion; green mark, surgical procedure with infrarenal aorta occlusion; yellow arrows, 2 Hz SCS; blue arrows, intrathecal $\alpha$-BGT injection. $\alpha-B G T, \alpha$-Bungarotoxin; $C$, control. 
assignment. A viable $\alpha$-motor neuron was defined as a motor neuron with soma diameter of 30 to $60 \mu \mathrm{m}$ and basophilic striping of the Nissl body. ${ }^{17}$ Three sections from each rabbit were examined, and an average was calculated.

\section{TUNEL Staining}

In situ TUNEL staining for detection of apoptotic DNA, fragmentation, identification, and quantification of apoptotic motor neurons was performed according to the manufacturer's instruction (Roche Diagnostics, Mannheim, Germany). TUNEL-positive motor neurons were defined as morphologically normal neurons with brown nuclei under a light microscope. Three sections from each rabbit were stained. The numbers of TUNEL-positive motor neurons and total motor neurons were counted by a trained observer who was blinded to the section grouping. The rate of TUNEL-positive motor neurons was defined as the average percentage of motor neurons that was TUNEL-positive.

\section{Microglia Staining}

Ionized calcium binding adaptor molecules 1 (Iba1), which express on the surface of microglia,${ }^{18}$ were stained to enable the visualization of microglia under light microscope, and thus enable the observation of microglia activation that manifests as microglia population expansion and morphology transformation ${ }^{19,20}$ (cellular body enlarged, processes thickened, ramified microglia transformed to ameboid form). The staining method was as follows: Paraffin-embedded spinal cord sections were deparaffinized and rehydrated. High-pressure steam for 2 minutes with ethylenediaminetetraacetic acid $(\mathrm{pH}$ 8.0) was used for antigen retrieval. Endogenous peroxidase activity was quenched with $3 \% \mathrm{H}_{2} \mathrm{O}_{2}$. After washing with phosphate-buffered saline, the sections were incubated overnight at $4{ }^{\circ} \mathrm{C}$ with goat polyclonal anti-Iba1 antibody (Abcam, Cambridge, UK) at a dilution of 1:3000. The next day, the sections were rewarmed for 1 hour at room temperature before rinsing in phosphatebuffered saline 3 times and then incubated 2 hours at room temperature with a second antibody (polyclonal anti-goat immunoglobulin-G horseradish peroxidase, Boster Biological Technology, Wuhan, China). Diaminobenzidine was used as chromogen, and all slides were counterstained with hematoxylin. Ventral horn images of all sections were taken under light microscope at $100 \times$ magnification. The ratio of the Iba1-stained area in the spinal cord anterior horn to the anterior horn area and the microglia area ratio of each section was analyzed using Image-Pro Plus6.0 by an observer, who was blinded to the section grouping. Three sections from each rabbit were stained, and an average value of microglia area ratio was calculated.
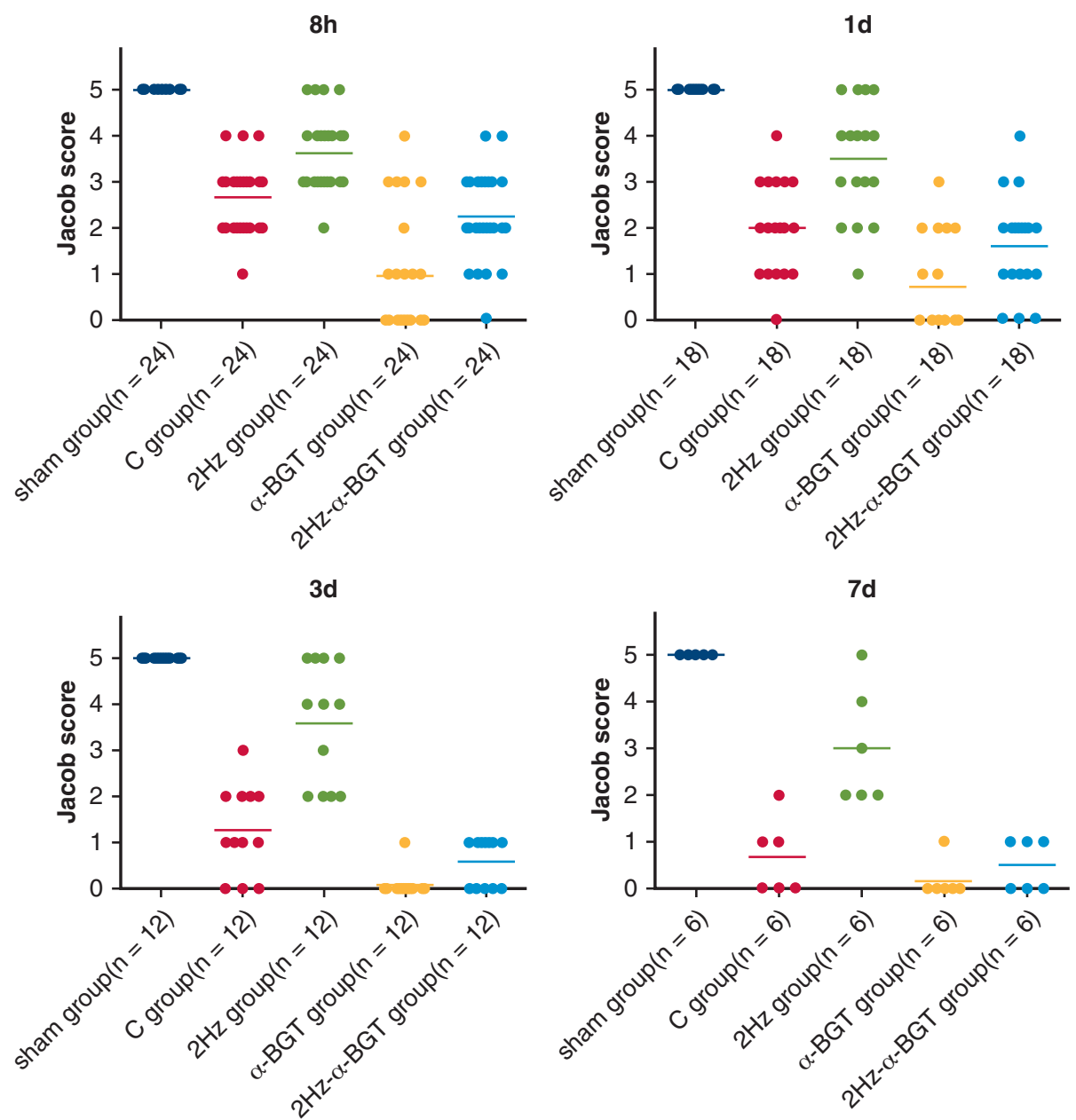

FIGURE 2. Hind-limb neurologic evaluation of rabbits. Dot plots were used to present the distribution of rabbits' Jacob scores of each group at 8 hours, 1 day, 3 days, and 7 days of spinal cord reperfusion with the horizontal bar representing the median Jacob score of the group. Blue dots, sham group; red dots, control group; green dots, $2 \mathrm{~Hz}$; yellow dots, $\alpha$-BGT group; cyan dots, $2 \mathrm{~Hz}-\alpha$-BGT group. $\alpha$-BGT, $\alpha$-Bungarotoxin; $C$, control. 


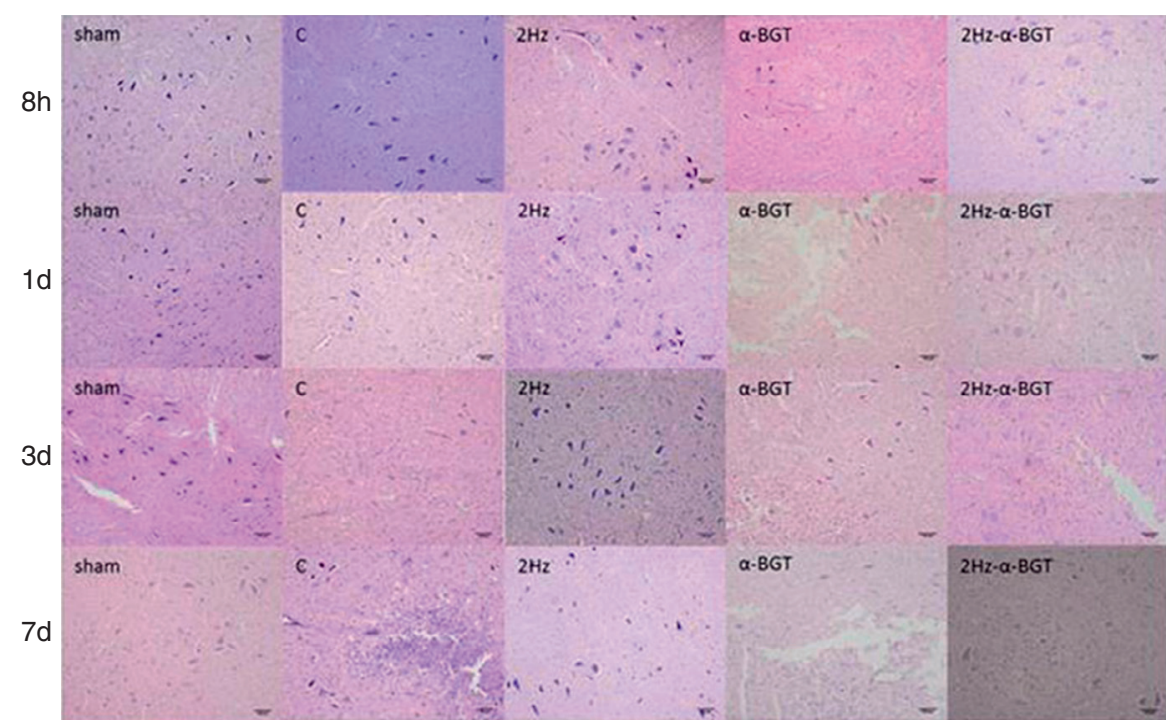

A
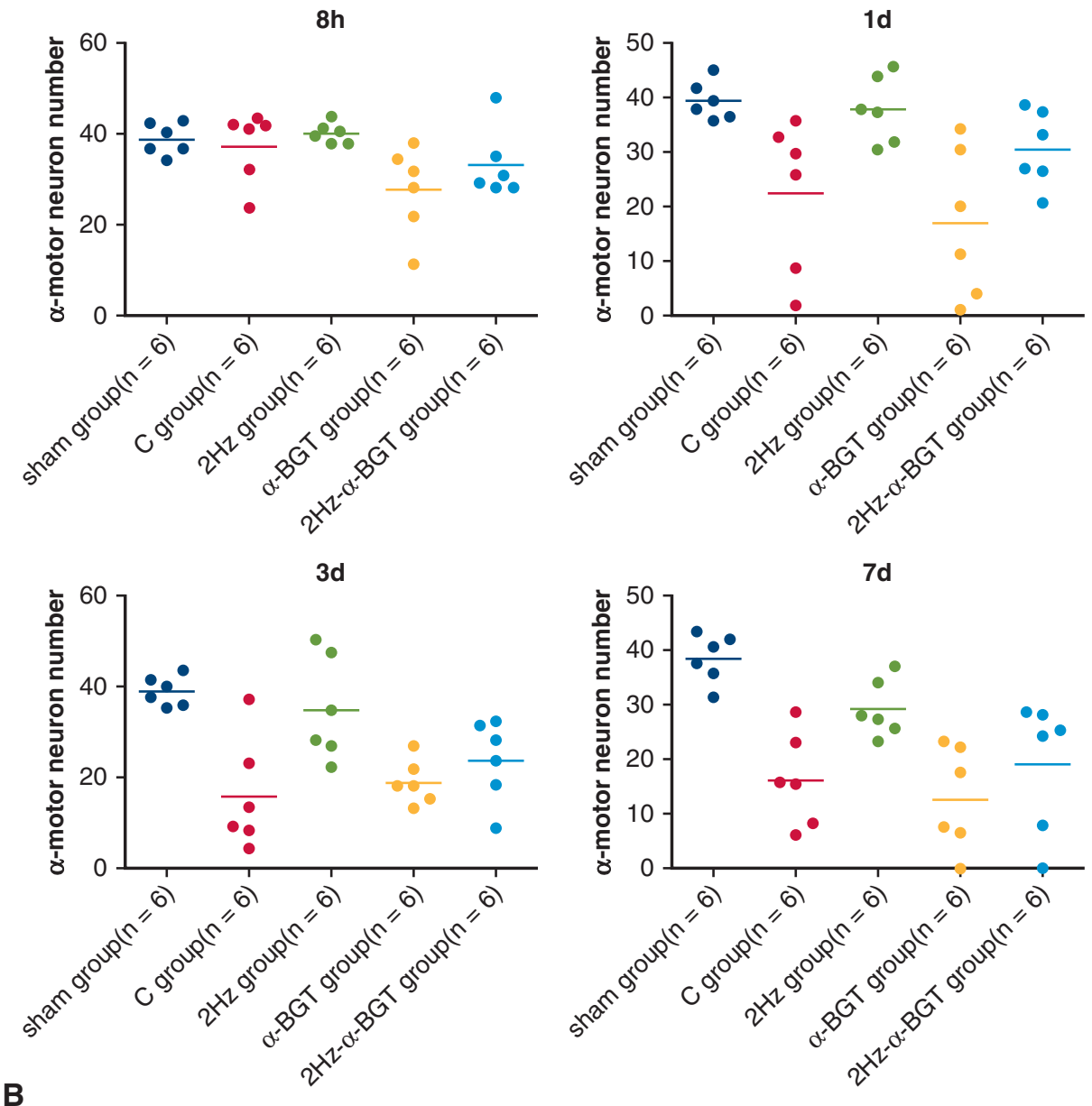

FIGURE 3. Histologic evaluation of spinal cord. A, Representative sections of lumber spinal cords stained with HE. Original magnification, $\times 100$. B, Distribution of spinal cord $\alpha$-motor neuron number of each group at 8 hours, 1 day, 3 days, and 7 days of spinal cord reperfusion. Data presented as dot plots: Blue dots represent sham group, red dots represent control group, green dots represent $2 \mathrm{~Hz}$ group, yellow dots represent $\alpha$-BGT group, and cyan dots represent $2 \mathrm{~Hz}-\alpha$-BGT group. The horizontal bar represents the median number of viable $\alpha$-motor neurons of the group. $\alpha$-BGT, $\alpha$-Bungarotoxin; $C$, control. 


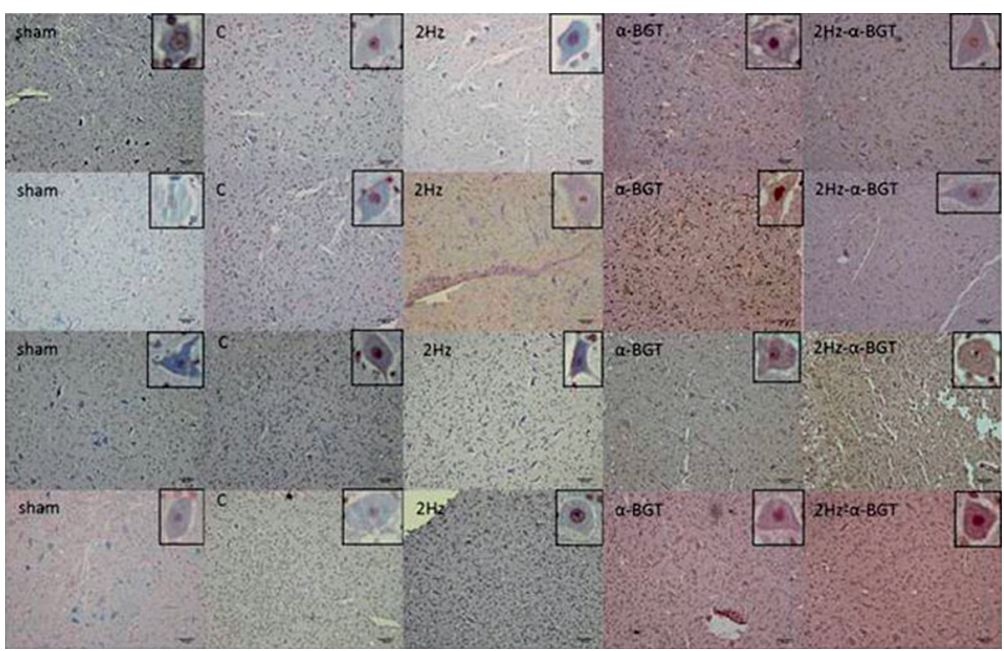

A
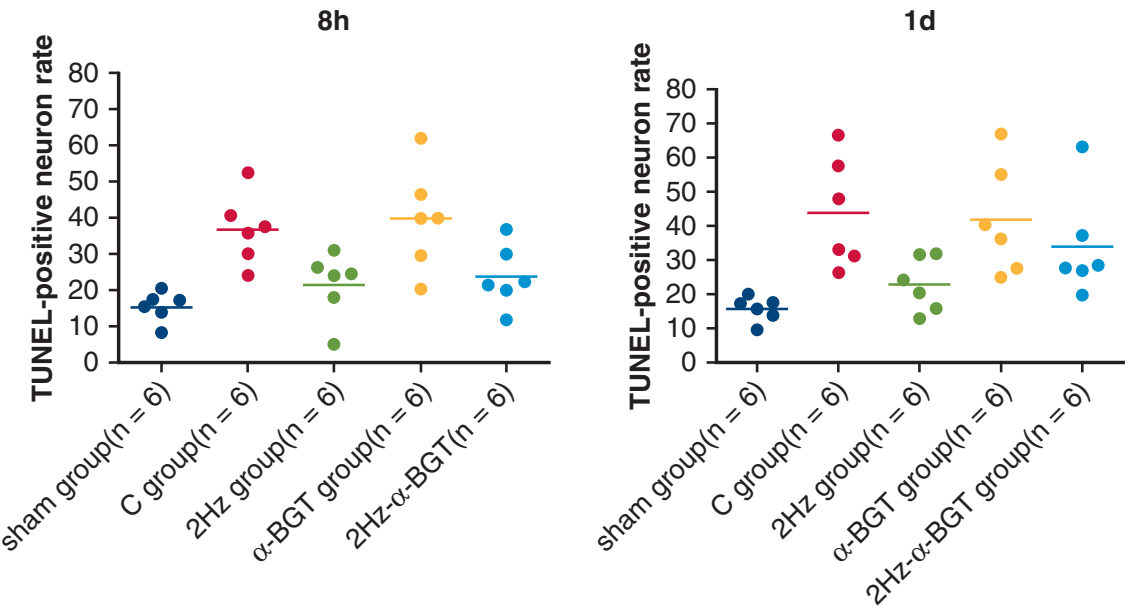

3d
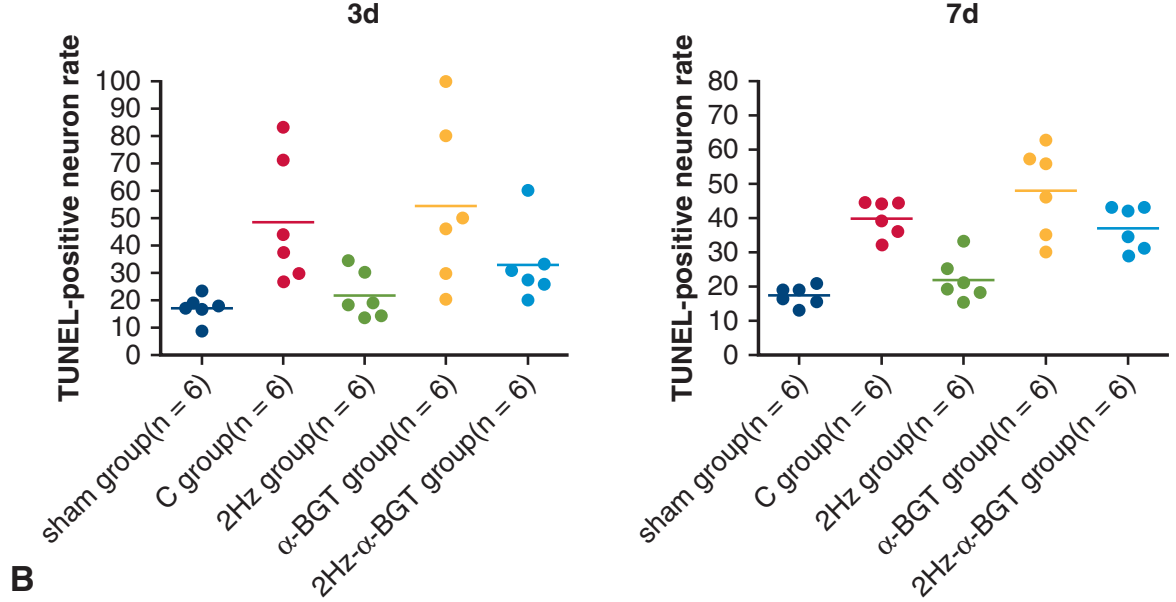

FIGURE 4. TUNEL staining of spinal cord of rabbits in sham, control, $2 \mathrm{~Hz}, \alpha$-BGT, and $2 \mathrm{~Hz}-\alpha$-BGT groups at 8 hours, 1 day, 3 days, and 7 days of spinal cord reperfusion. A, Representative TUNEL-stained sections of lumber spinal cords with a magnified example of a TUNEL-positive $\alpha$-motor neuron in each section. Original magnification, $\times 100$. B, Distribution of TUNEL-positive $\alpha$-motor neuron rates. Data presented as dot plots: blue dots represent sham group, red dots represent the control group, green dots represent $2 \mathrm{~Hz}$ group, yellow dots represent $\alpha$-BGT group, and cyan dots represent $2 \mathrm{~Hz}-\alpha$-BGT group. The horizontal bar represents the median rate of TUNEL-positive $\alpha$-motor neuron of the group. $\alpha-B G T$, $\alpha$-Bungarotoxin; $C$, control; TUNEL, terminal deoxynucleotidyl transferase-mediated dUTP nick end labeling. 


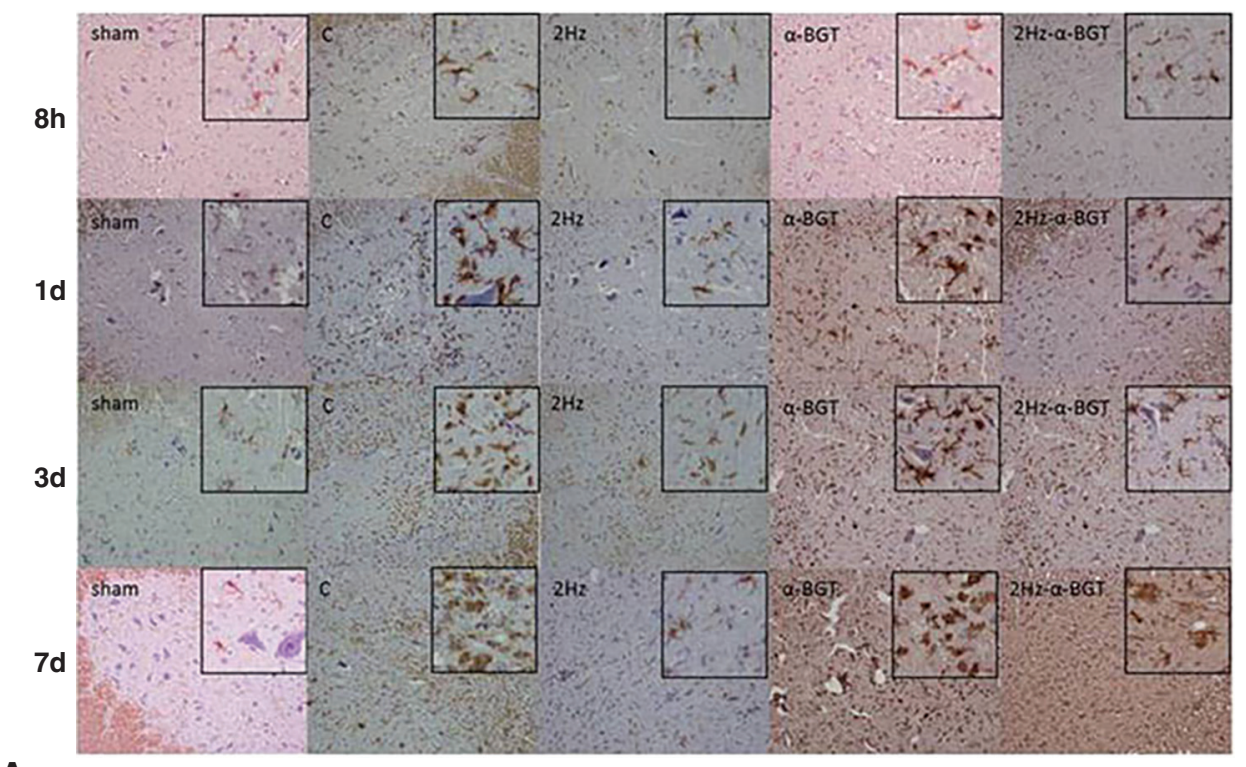

A
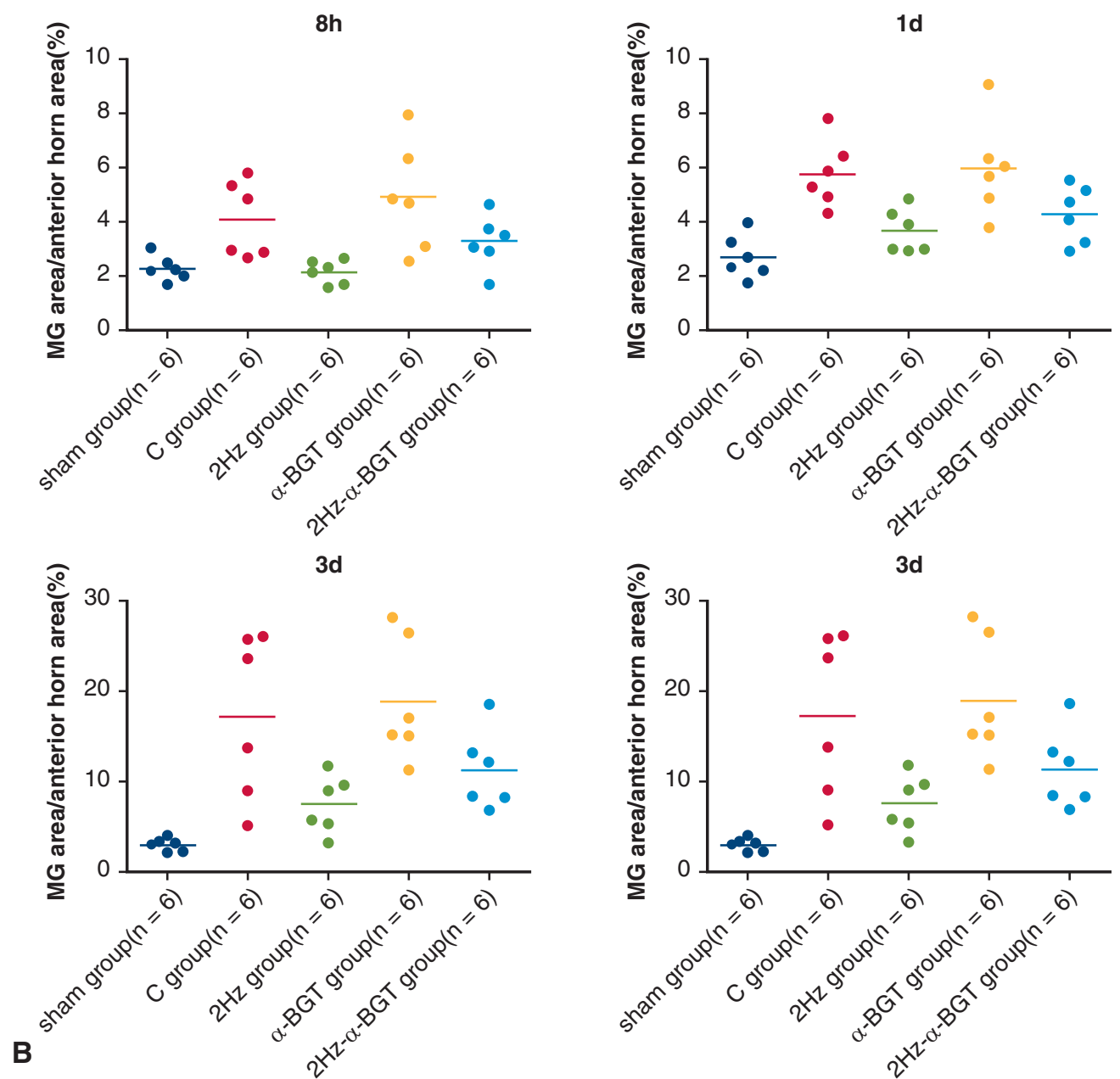

FIGURE 5. Microglia staining of spinal cord ventral horns of rabbits in sham, control, $2 \mathrm{~Hz}, \alpha$-BGT, and $2 \mathrm{~Hz}-\alpha$-BGT groups at 8 hours, 1 day, 3 days, and 7 days of reperfusion. A, Representative microglia-stained sections of lumber spinal cord. Original magnification, $\times 100$. B, Distribution of ratios of microglia-stained area to anterior horn area. Data presented as dots plots: blue dots represent sham group, red dots represent the control group, green dots represent $2 \mathrm{~Hz}$ group, yellow dots represent $\alpha$-BGT group, and cyan dots represent $2 \mathrm{~Hz}-\alpha$-BGT group. The horizontal bar represents the median ratio of microglia-stained area to anterior horn area of the group. $\alpha$-BGT, $\alpha$-Bungarotoxin; $C$, control; $M G$, microglia. 


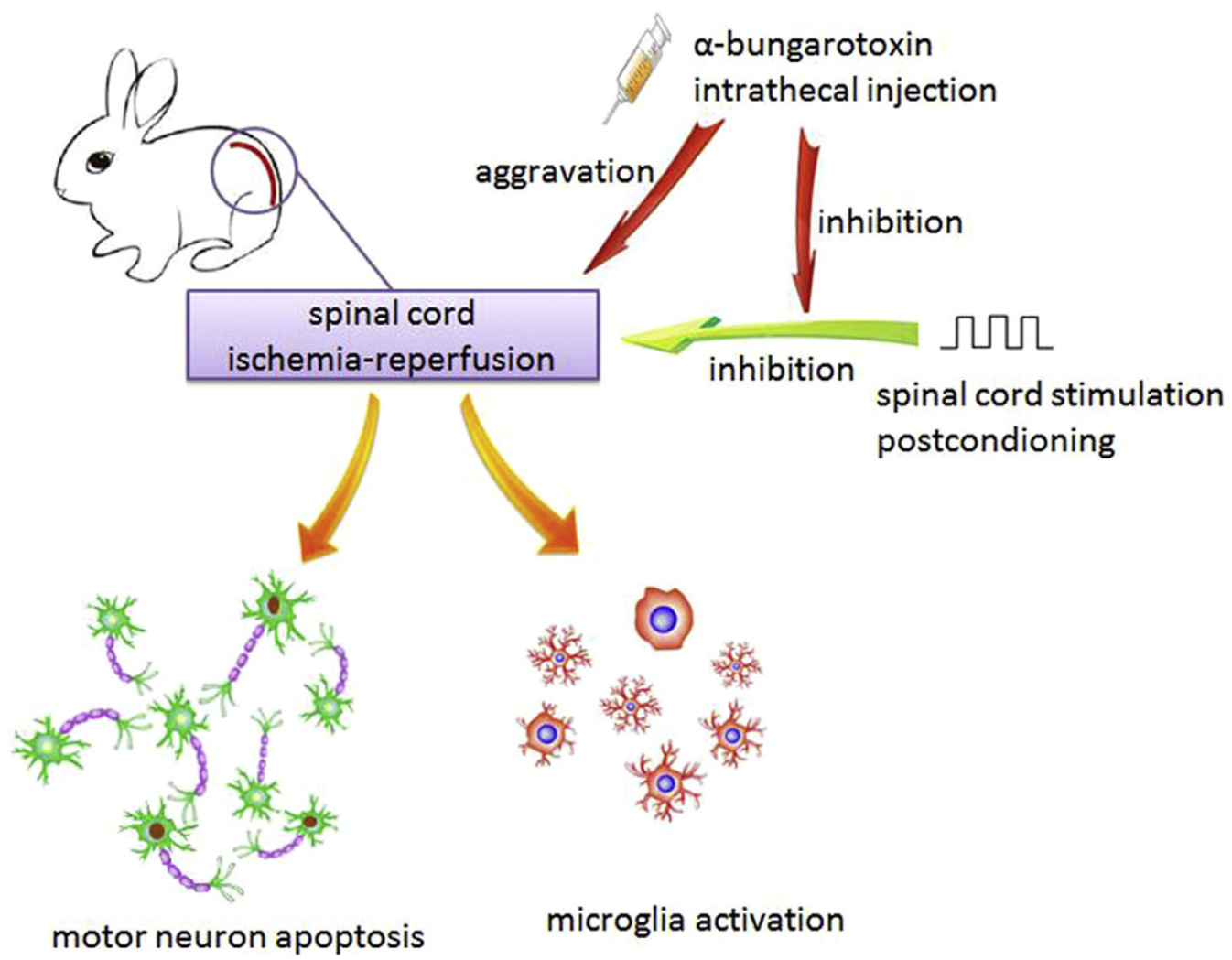

FIGURE 6. Neuroprotective mechanism involved in SCS postconditioning; $2 \mathrm{~Hz}$ SCS postconditioning inhibited spinal cord I/R injury through microglia inhibition, the mechanism of which was related to activation of $\alpha 7 \mathrm{nAchRs}$ because antagonizing $\alpha 7 \mathrm{nAchRs}$ by injecting $\alpha$-BGT (a specific $\alpha 7 \mathrm{nAchR}$ antagonist) intrathecally partially suppressed the neuroprotective effects of SCS postconditioning.

\section{Statistical Analysis}

A multilevel cumulative logistic regression model with rabbit as a random effect was used to analyze the neurologic scores. In the mixedeffects model, 4 "dummy" binary variables with the control group or sham group as reference group, time, and the interactions between time and the dummy variables were included. A linear or nonlinear trend (log or power function) of time was first fitted to the ordinal neurologic scores, and then the best function of time was included in the final mixed model. Quantile regression was applied to the analysis of numbers of viable $\alpha$-motor neurons, TUNEL-positive motor neuron rates, and microglia area ratios after the trend of the time was first tested. Logit transformation was performed on the data of TUNEL-positive motor neuron rates and microglia area ratios. Statistical analyses were performed using $\mathrm{R}$ version 3.4.1 and SPSS Statistics 25.0 (SPSS Inc, Chicago, Ill). Figures 1 to 6 were drawn by using Adobe Illustrator CC version 2014 (San Jose, Calif) and GraphPad Prism 5 version 5.0 (GraphPad Software, San Diego, Calif).

\section{RESULTS}

A total of 124 rabbits were used, and 4 rabbits were excluded from the experiment preischemia because of neurologic impairment of the hind limb induced by implantation of the stimulation electrode/PE tube. Results in the control group as the reference group were as follows, and the results in the sham group as the reference group are shown in the Tables E1 and E2.

\section{Neurologic Function}

There were no time effects in the Jacob scores of the sham group and $2 \mathrm{~Hz}$ group by preanalysis, which could be seen in the distribution of Jacob scores in these 2 groups at prescribed time points (Figure 2). The time (t) function

TABLE 1. Mixed-effect ordinal logistic regression analysis of Jacob scores in relation to grouping and time (compared with control group)

\begin{tabular}{lccc}
\hline \multicolumn{1}{c}{ Group } & Coefficient & $\boldsymbol{P}$ value & $\mathbf{9 5 \%} \mathbf{C I}$ \\
\hline Control & Ref. & - & - \\
\hline Sham & 5.60 & $<.001$ & 3.80 to 7.40 \\
\hline $2 \mathrm{~Hz}$ & 1.69 & $<.001$ & 0.09 to 2.52 \\
$2 \mathrm{~Hz}-\alpha$-BGT & 0.34 & .407 & -0.46 to 1.14 \\
$\alpha-\mathrm{BGT}$ & -1.48 & $<.001$ & -2.34 to -0.63 \\
$\mathrm{t}^{0.4}$ & & & \\
\hline Sham $* \mathrm{t}^{0.4}$ & -0.00 & 1.000 & -0.45 to 0.45 \\
$2 \mathrm{~Hz} \mathrm{t}^{0.4}$ & -0.01 & .936 & -0.18 to 0.17 \\
\hline $2 \mathrm{~Hz}-\alpha-\mathrm{BGT} * \mathrm{t}^{0.4}$ & -0.27 & .004 & -0.45 to -0.09 \\
$\alpha-\mathrm{BGT}^{*} \mathrm{t}^{0.4}$ & -0.28 & .019 & -0.51 to -0.05 \\
\hline
\end{tabular}

CI, Confidence interval; $\alpha$-BGT, $\alpha$-bungarotoxin. 
TABLE 2. Mixed-effect ordinal logistic regression analysis of $\alpha$-motor neuron numbers, rates of TUNEL-positive motor neurons, and ratios of microglia area/anterior horn area in relation to grouping and time (compared with control group)

\begin{tabular}{|c|c|c|c|c|c|c|}
\hline \multirow[b]{2}{*}{ Group } & \multicolumn{2}{|c|}{ Numbers of $\alpha$-motor neuron } & \multicolumn{2}{|c|}{$\begin{array}{c}\log (\text { TUNEL-positive/1- } \\
\text { TUNEL-positive motor neuron rate) }\end{array}$} & \multicolumn{2}{|c|}{$\begin{array}{l}\log (\text { microglia area ratio/ } \\
\text { 1-microglia area ratio) }\end{array}$} \\
\hline & Coefficient & $95 \%$ CI & Coefficient & $95 \% \mathrm{CI}$ & Coefficient & $95 \%$ CI \\
\hline Control (Ref) & 23.0 & 15.2 to 29.9 & -0.51 & -0.59 to -0.23 & -2.67 & -2.87 to -1.61 \\
\hline Sham & 14.7 & 8.9 to 24.6 & -1.05 & -1.36 to -0.97 & -0.81 & -1.79 to -0.65 \\
\hline $2 \mathrm{~Hz}$ & 14.0 & 5.8 to 21.2 & -0.81 & -1.19 to -0.56 & -0.42 & -1.52 to -0.09 \\
\hline $2 \mathrm{~Hz}-\alpha-\mathrm{BGT}$ & 5.0 & -1.5 to 12.1 & -0.40 & -0.70 to -0.23 & -0.15 & -1.10 to 0.41 \\
\hline$\alpha$-BGT & -3.0 & -10.5 to 5.5 & 0.13 & -0.27 to 0.44 & 0.37 & -0.79 to 1.05 \\
\hline
\end{tabular}

TUNEL, Terminal deoxynucleotidyl transferase-mediated dUTP nick end labeling; $C I$, confidence interval; $\alpha$-BGT, $\alpha$-bungarotoxin.

best fitted for Jacob scores of the control, $\alpha$-BGT, and $2 \mathrm{~Hz}$ $\alpha$-BGT groups was time to the power of $0.4\left(\mathrm{t}^{0.4}\right)$. Therefore, only 4 "dummy" binary variables representing the groups and the interaction between time and binary variables were included in the final mixed-effect ordinal logistic regression analysis (Table 1). As shown in Table 1 and Figure 2, the Jacob scores of the sham and $2 \mathrm{~Hz}$ groups were significantly higher than in the control group $(P<.001)$. Conversely, the scores of the $\alpha$-BGT group were significantly lower than in the control group $(P<.001)$. The Jacob scores of the $2 \mathrm{~Hz}-\alpha$-BGT group were not significantly different from those of the control group $(P=.407)$. There were no time and grouping interactions in the Jacob score of the sham and $2 \mathrm{~Hz}$ groups ( $P=1.000$ and .936 , respectively), whereas there were significant decreases of Jacob scores with the increase of time in the $2 \mathrm{~Hz}-\alpha$-BGT group and the $\alpha$-BGT group $(P=.004$ and .019 , respectively). The same time trends of Jacob scores among the control, $2 \mathrm{~Hz}-\alpha$-BGT, and $\alpha$-BGT groups could be seen when the sham group acted as the reference group (Table E1).

\section{Histologic Assessment}

Representative images of HE staining and distribution of viable $\alpha$-motor neuron numbers of each group at prescribed time points are shown in Figure 3. When a quantile regression was applied to the analysis of numbers of $\alpha$-motor neurons with different power of time, the smallest sum of the absolute residuals was obtained when the power approximately equaled zero. Thus, only 4 "dummy" binary variables were included in the final quantile regression for the numbers of $\alpha$-motor neurons. As shown in Table 2, the numbers of $\alpha$-motor neurons of the sham and $2 \mathrm{~Hz}$ groups were significantly higher than those of the control group, the reference group, with 14.7 (95\% confidence interval $[\mathrm{CI}], 8.9-24.6)$ and $14.0(95 \% \mathrm{CI}, 5.8-21.2)$, respectively. The numbers of $\alpha$-motor neurons in the 2 $\mathrm{Hz}-\alpha$-BGT and $\alpha$-BGT groups were not significantly different from those of the control group. Table E2 shows results when the sham group acted as the reference group.

\section{TUNEL Staining}

Representative images of TUNEL staining and distribution of TUNEL-positive motor neuron rates of each group at prescribed time points are shown in Figure 4. A similar result of time trend in the analysis of $\alpha$-motor neuron numbers was obtained in the analysis of rates of TUNEL-positive motor neurons. Quantile regression results are shown in Table 2. The logits of the rates of TUNEL-positive motor neurons in the sham, $2 \mathrm{~Hz}$, and $2 \mathrm{~Hz}-\alpha$-BGT groups were significantly lower than those of the control group, the reference group with -1.05 (95\% CI, -1.36 to -0.97$),-0.81$ (95\% CI, -1.19 to -0.56$)$, and $-0.40(95 \% \mathrm{CI},-1.36$ to -0.97$)$, respectively (Table 2). The logits of the rates of TUNEL-positive motor neurons in the $\alpha$-BGT group were not significantly different from those of the control group. Table E2 showes results when the sham group acted as reference group.

\section{Microglia Staining}

Figure 5 shows representative Iba1 staining sections and distribution of microglia area ratios of each group at prescribed time points. The similar time trend result in the analysis of $\alpha$-motor neuron numbers was obtained in the analysis of the microglia area ratios. As shown in Table 2, the logits of microglia area ratios in the sham and $2 \mathrm{~Hz}$ groups were significantly lower than those of the control group, the reference group with $-0.81(95 \%$ CI, -1.79 to -0.65$)$ and $-0.42(95 \% \mathrm{CI},-1.52$ to $-0.09)$, respectively. The logits of microglia area ratios in the $2 \mathrm{~Hz}-\alpha$-BGT and $\alpha$-BGT groups were not significantly different from those of the control group. Table E2 shows results when the sham group acted as the reference group.

\section{DISCUSSION}

The current study investigated whether $\alpha 7 \mathrm{nAchRs}$ were involved in the neuroprotective effects of $2 \mathrm{~Hz}$ SCS postconditioning in spinal cord $\mathrm{I} / \mathrm{R}$ injury. By using $\alpha 7 \mathrm{nAchR}$ antagonist, $\alpha$-BGT, the current study demonstrated that the neuroprotective effects of $2 \mathrm{~Hz}$ SCS 
were related to $\alpha 7 \mathrm{nAchR}$-mediated microglia inhibition and that $\alpha 7 \mathrm{nAchR}$ antagonist partially inhibited the neuroprotective effects of $2 \mathrm{~Hz}$ SCS postconditioning.

In the current study, $2 \mathrm{~Hz}$ SCS postconditioning significantly inhibited spinal cord microglia activation induced by I/R injury, as shown in Figure 5 and Table 2; the microglia area ratios of the $2 \mathrm{~Hz}$ group were significantly lower than in the control group at all prescribed time points. Consistent with previous studies, ${ }^{8-10}$ the current study demonstrated that microglia inhibition enhances neuronal survival and improves neurologic outcomes, which was presented compared with the control group, and rabbits in the $2 \mathrm{~Hz}$ group preserved significantly better neurologic functions, more $\alpha$-motor neurons, and lower TUNEL-positive neuron rates.

As for the mechanism of microglia inhibition, previous studies suggested that a number of drugs and methods exerted neuroprotective effects through $\alpha 7 \mathrm{nAchR}$-mediated microglia inhibition. As one of the most abundant nAchR subtypes in the central nervous system, $\alpha 7 \mathrm{nAchR}$ is widely expressed on the membrane surface of neurons, microglia, and endothelial cells in the brain and spinal cord, and related to a variety of central nervous system diseases. ${ }^{21}$ In ischemia insult, $\alpha 7 \mathrm{nAchR}$ activation improves neuronal survival. For example, Rosa and colleagues ${ }^{22}$ demonstrated that nicotine decreased lactate dehydrogenase release of hippocampal slices subjected to oxygen-glucose deprivation plus reperfusion in wild-type mice, but failed to exert this neuroprotective effect in $\alpha 7 \mathrm{nAchR}$ knockout mice, and the neuroprotective effect of nicotine was antagonized by the nonselective nicotinic receptor blocker mecamylamine. In vivo studies further suggest that $\alpha 7$ nAchR activation mediates microglia inhibition, thus decreasing the release of proinflammatory cytokines by microglia, suppresses detrimental inflammatory responses, enhances neuronal survival, and improves neurologic outcome. By using cerebral ischemic animal models, Guan and colleagues, ${ }^{13}$ Parada and colleagues, ${ }^{11}$ and Kalappa and colleagues ${ }^{23}$ demonstrated that administration of nicotine or PNU282987 (a synthetic $\alpha 7 \mathrm{nAchR}$ agonist) or PNU120596 (a type II positive allosteric modulator of $\alpha 7 \mathrm{nAchR}$ ) significantly reduced CA1 pyramidal neuron death and microglia activation and proliferation, and decreased brain infarct volume. In addition to pharmacologic manipulation of $\alpha 7 \mathrm{nAchRs}$, methods that have been studied widely in central nervous system I/R injury also exert neuroprotective effects via $\alpha 7 \mathrm{nAchR}$ activation. Wang and colleagues ${ }^{24}$ demonstrated that electroacupuncture pretreatment prevented the reduction of $\alpha 7 \mathrm{nAchR}$ expression in rats' ischemic penumbra, reduced brain infarct volume, and improved neurologic function. Moreover, these neuroprotective effects were attenuated by $\alpha$-BGT. To investigate whether $\alpha 7 \mathrm{nAchRs}$ were also involved in the microglia inhibition of $2 \mathrm{~Hz}$ SCS postconditioning, we injected $\alpha$-BGT, a specific $\alpha 7 \mathrm{nAchR}$ antagonist, into the intrathecal space of rabbits before $2 \mathrm{~Hz}$ SCS in the $2 \mathrm{~Hz}-\alpha$-BGT group and demonstrated that the neuroprotective effects of $2 \mathrm{~Hz}$ SCS were partially inhibited. As shown in Tables 1 and 2, the hind-limb Jacob scores, numbers of $\alpha$-motor neurons, and microglia area ratios of the $2 \mathrm{~Hz}-\alpha$-BGT group were not significantly different from those of the control group. Only the TUNEL-positive motor neuron rates were significantly lower than those of the control group. Thus, as summarized in Figure 6, our study demonstrated that $2 \mathrm{~Hz}$ SCS postconditioning inhibited spinal cord I/R injury through microglia inhibition, the mechanism of which was related to activation of $\alpha 7 \mathrm{nAchRs}$ because antagonizing $\alpha 7 \mathrm{nAchRs}$ partially suppressed the neuroprotective effects of $2 \mathrm{~Hz}$ SCS postconditioning.

To the best of our knowledge, this is the first study linking $\alpha 7$ nAchRs to neuroprotective effects of SCS postconditioning. This expands our understanding of the mechanism of SCS; however, how $2 \mathrm{~Hz}$ SCS postconditioning acts via $\alpha 7$ nAchRs is unknown. There are several possibilities. First, $2 \mathrm{~Hz}$ SCS postconditioning may link to $\alpha 7 \mathrm{nAchRs}$ through cholinergic anti-inflammatory pathway. Previous studies of sepsis ${ }^{25}$ and diseases with a strong inflammatory component, such as rheumatoid arthritis ${ }^{26}$ and $\mathrm{I} / \mathrm{R},{ }^{14,15,27}$ suggested that vagus nerve stimulation exerts an antiinflammatory effect via $\alpha 7 \mathrm{nAchRs}$, leading to the discovery of the cholinergic anti-inflammatory pathway. Further studies have confirmed that $\alpha 7 \mathrm{nAchRs}$ are required for the functional integrity of the cholinergic antiinflammatory pathway. In the cholinergic antiinflammatory pathway, peripheral information is conveyed to the nucleus tractus solitarius of the brain by afferent fibers of vagus nerve and then delivered to the dorsal motor nucleus of vagus. In the dorsal motor nucleus of vagus, efferent fibers of vagus nerve are stimulated. ${ }^{28}$ Acetylcholine is released from synaptic terminals of the stimulated efferent vagus nerve and binds to $\alpha 7 \mathrm{nAchRs}$ on immune cells, and thus proinflammatory cytokine release is inhibited. In this process, through neuro-projections, modulations from forebrain sites, including hypothalamic nuclei, rostral ventrolateral medulla, and locus coeruleus, are involved. ${ }^{29}$ In SCS analgesia, a comprehensive set of studies $^{30,31}$ indicate that supraspinal structures such as neural circuits in the brainstem, anterior pretectal nucleus, and locus coeruleus are involved, so it is possible that SCS links to cholinergic anti-inflammatory pathway through brain nuclei projections. Second, $2 \mathrm{~Hz}$ SCS postconditioning may link to $\alpha 7 \mathrm{nAchRs}$ by preventing reduction of $\alpha 7 \mathrm{nAchRs}$ expression in the spinal cord after $\mathrm{I} / \mathrm{R}$ injury. By using a rat model of transient cerebral ischemia, Wang and colleagues $^{32}$ demonstrated that $\alpha 7 \mathrm{nAchR}$ 
expression was significantly decreased in the ischemic penumbras, whereas electroacupuncture pretreatment at Baihui (GV 20) inhibited this reduction and attenuated cerebral ischemic injury. Third, $2 \mathrm{~Hz}$ SCS postconditioning may link to $\alpha 7 \mathrm{nAchRs}$ by increasing $\alpha 7 \mathrm{nAchR}$ expression in the spinal cord after I/R injury. In contrast to Wang and colleagues' study, ${ }^{32}$ Liu and colleagues ${ }^{33}$ demonstrated that $\alpha 7 \mathrm{nAchR}$ expression increased mildly 12 hours after cerebral ischemia and puerarin pretreatment increased $\alpha 7 \mathrm{nAchR}$ expression significantly, reduced infarct volume, and improved neurologic outcome. Although the mechanism of the neuroprotective effects of SCS still requires further study, the encouraging results of the current study suggest that SCS postconditioning could be a potential option to protect the spinal cord from I/R injury in clinical practice.

\section{Study Limitations}

One limitation of the current study is that we neglected to include an $\alpha$-BGT control group (rabbits with intrathecal injection of $\alpha$-BGT but without abdominal aorta occlusion) to better define the specific impact of the introduction of $\alpha$-BGT into the intrathecal space.

\section{CONCLUSIONS}

The current study demonstrates that the neuroprotective effects of $2 \mathrm{~Hz}$ SCS in spinal cord I/R injury are related in part to activation of $\alpha 7 \mathrm{nAchRs}$. To the best of our knowledge, this is the first study that links the neuroprotective effect of SCS to $\alpha 7 \mathrm{nAchRs}$. However, further studies are needed to elucidate the exact mechanism linking SCS to $\alpha 7$ nAchRs.

\section{Conflict of Interest Statement}

Authors have nothing to disclose with regard to commercial support.

\section{References}

1. Coselli JS, LeMaire SA, Conklin LD, Koksoy C, Schmittling ZC. Morbidity and mortality after extent II thoracoabdominal aortic aneurysm repair. Ann Thorac Surg. 2002; 73:1107-15.

2. Li HX, Dong XH, Jin M, Cheng WP. The protective effect of spinal cord stimulation postconditioning against spinal cord ischemia/reperfusion injury in rabbits. Neuromodulation. 2018;21:448-56.

3. Dong XH, Li HX, Lu JK, Yang YW, Jing H, Cheng Y, et al. Spinal cord stimulation postconditioning reduces microglial activation through downregulation of ERK1/2 phosphorylation during spinal cord ischemic reperfusion in rabbits. Neuroreport. 2018;29:1180-7.

4. Olson JK. Immune response by microglia in the spinal cord. Ann NY Acad Sci. 2010;1198:271-8

5. Matsumoto S, Matsumoto M, Yamashita A, Ohtake K, Ishida K, Morimoto Y, et al. The temporal profile of the reaction of microglia, astrocytes, and macrophages in the delayed onset paraplegia after transient spinal cord ischemia in rabbits. Anesth Analg. 2003;96:1777-84.

6. Smith PD, Puskas F, Meng X, Lee JH, Cleveland JC Jr, Weyant MJ, et al. The evolution of chemokine release supports a bimodal mechanism of spinal cord ischemia and reperfusion injury. Circulation. 2012;126(Suppl 1):110-7.

7. Nakata T, Kawachi K, Nagashima M, Yasugi T, Izutani H, Ryugo M, et al. Transient ischemia-induced paresis and complete paraplegia displayed distinct reactions of microglia and macrophages. Brain Res. 2011;1420 114-24.

8. Tikka T, Fiebich BL, Goldsteins G, Keinanen R, Koistinaho J. Minocycline, a tetracycline derivative, is neuroprotective against excitotoxicity by inhibiting activation and proliferation of microglia. J Neurosci. 2001;21:2580-8.

9. Smith PD, Bell MT, Puskas F, Meng X, Clevrlang JC Jr, Weyant MJ, et al. Preservation of motor function after spinal cord ischemia and reperfusion injury through microglial inhibition. Ann Thorac Surg. 2013; 95:1647-53.

10. Takeda M, Kawaguchi M, Kumatoriya T, Horiuchi T, Watanabe K, Inoue S, et al Effects of minocycline on hind-limb motor function and gray and white matter injury after spinal cord ischemia in rats. Spine. 2011;36:1919-24.

11. Parada E, Egea J, Buendia I, Negredo P, Cunha AC, Cardoso S, et al. The microglial $\alpha 7$-acetylcholine nicotinic receptor is a key element in promoting neuroprotection by inducing heme oxygenase-1 via nuclear factor erythroid-2-related factor 2. Antioxid Redox Signal. 2013;194:1135-48.

12. Han ZY, Shen FX, He Y, Degos V, Camus M, Maze M, et al. Activation of a-7 nicotinic acetylcholine receptor reduces ischemic stroke injury through reduction of pro-inflammatory macrophages and oxidative stress. PLoS One. 2014;9: e105711.

13. Guan YZ, Jin XD, Guan LX, Yan HC, Wang P, Gong Z, et al. Nicotine inhibits microglial proliferation and is neuroprotective in global ischemia rats. $\mathrm{Mol} \mathrm{Neu}$ robiol. 2014;51:1480-8.

14. Sun Z, Baker W, Hiraki T, Greenberg JH. The effect of right vagus nerve stimulation on focal cerebral ischemia: an experimental study in the rat. Brain Stimul. 2012;5:1-10.

15. Jiang Y, Li L, Liu B, Zhang Y, Chen Q, Li C. Vagus nerve stimulation attenuates cerebral ischemia and reperfusion injury via endogenous cholinergic pathway in rat. PLoS One. 2014;9:e102342.

16. Jacobs TP, Schohami E, Baze W, Burgard E, Gunderson C, Hallenbeck JM, et al Deteriorating stroke model: histopathology, edema and eicosanoid changes following spinal cord ischemia in rabbits. Stroke. 1987;18:741-50.

17. Huang H, Zhang L, Wang Y, Yao J, Weng H, Wu H, et al. Effect of ischemic postconditioning on spinal cord ischemic-reperfusion injury in rabbits. Can J Anesth 2007; 54:42-8

18. Ito D, Imai Y, Ohsawa K, Nakajima K, Fukuuchi Y, Kohsaka S. Microglia-specific localisation of a novel calcium binding protein, Iba1. Brain Res Mol Brain Res. 1998;57:1-9.

19. Ajami B, Bennett JL, Krieger C, Tetzlaff W, Rossi FM. Local self-renewal can sustain CNS microglia maintenance and function throughout adult life. $\mathrm{Nat} \mathrm{Neu}$ rosci. 2007;10:1538-43.

20. Ladeby R, Wirenfeldta M, Garcia-Ovejerob D, Fengera C, Dissing-Olesena L, Dalmaua I, et al. Microglial cell population dynamics in the injured adult central nervous system. Brain Res Rev. 2005;48:196-206.

21. Gotti C, Clementi F. Neuronal nicotinic receptors: from structure to pathology Prog Neurobiol. 2004; 74:363-96.

22. Rosa AO, Egea J, Gandia L, Lopez MG, Garcia AG. Neuroprotection by nicotine in hippocampal slices subjected to oxygen-glucose deprivation: involvement of the alpha7 nAChR subtype. J Mol Neurosci. 2006;30:61-2.

23. Kalappa BI, Sun F, Johnson SR, Jin K, Uteshev VV. A positive allosteric modulator of $\alpha 7 \mathrm{nAchRs}$ augments neuroprotective effects of endogenous nicotinic ag onists in cerebral ischemia. Br J Pharmacol. 2013;169:1862-78.

24. Wang H, Yu M, Ochani M, Amella CA, Tanovic M, Susarla S, et al. Nicotinic acetylcholine receptor alpha7 subunit is an essential regulator of inflammation. Nature. 2003;421:384-8.

25. Borovikova LV, Ivanova S, Zhang M, Yang H, Botchkina GI, Watkins LR, et al Vagus nerve stimulation attenuates the systemic inflammatory response to endotoxin. Nature. 2000;405:458-62.

26. Koopman FA, Schuurman PR, Vervoordeldonk MJ, Tak JJ. Vagus nerve stimulation: a new bioelectronics approach to treat rheumatoid arthritis? Best Pract Res Clin Rheumatol. 2014;28:625-35.

27. Zhang R, Wugeti N, Sun J, Yan H, Guo Y, Zhang L, et al. Effects of vagus nerve stimulation via cholinergic anti-inflammatory pathway activation on myocardial ischemia/reperfusion injury in canine. Int J Clin Exp Med. 2014; $7: 2615-23$

28. Tracey KJ. The inflammatory reflex. Nature. 2002;420:853-9.

29. Pavlov VA, Wang H, Czura CJ, Friedman SG, Tracey KJ. The cholinergic antiinflammatory pathway: a missing link in neuroimmunomodulation. Mol Med. 2003;9:125-34.

30. Tazawa T, Kamiya Y, Kobayashi A, Saeki K, Takiguchi M, Nakahashi Y, et al. Spinal cord stimulation modulates supraspinal centers of the 
descending antinociceptive system in rats with unilateral spinal nerve injury. Mol Pain. 2015;11:36.

31. Sivanesan E, Maher DP, Raja SN, Linderoth B, Guan Y. Supraspinal mechanisms of spinal cord stimulation for modulation of pain: five decades of research and prospects for the future. Anesthesiology. 2019;130: 651-65.

32. Wang Q, Wang F, Li X, Yang Q, Li X, Xu N, et al. Electroacupuncture pretreatment attenuates cerebral ischemic injury through a7 nicotinic acetylcholine receptor-mediated inhibition of high-mobility group box 1 release in rats. $\mathrm{J} \mathrm{Neu}$ roinflammation. 2012;9:24.
33. Liu X, Mei Z, Qian J, Zeng Y, Wang M. Puerarin partly counteracts the inflammatory response after cerebral ischemia/reperfusion via activating the cholinergic anti-inflammatory pathway. Neural Regen Res. 2013;8: 3203-15.

Key Words: spinal cord stimulation postconditioning, spinal cord ischemia/reperfusion injury, $\alpha 7$ nicotinic acetylcholine receptor, $\alpha$-bungarotoxin 
TABLE E1. Mixed-effect ordinal logistic regression analysis of Jacob scores in relation to grouping and time (compared with sham group)

\begin{tabular}{lccc}
\hline \multicolumn{1}{c}{ Group } & Coefficient & $\boldsymbol{P}$ value & $\mathbf{9 5 \%}$ CI \\
\hline Sham & Ref. & - & - \\
$2 \mathrm{~Hz}$ & -3.91 & $<.001$ & -5.27 to -2.56 \\
\hline $2 \mathrm{~Hz}-\alpha$-BGT & -5.33 & $<.001$ & -6.72 to -3.93 \\
Control & -4.83 & $<.001$ & -6.21 to -3.45 \\
\hline$\alpha$-BGT & -7.21 & $<.001$ & -8.67 to -5.74 \\
$\mathrm{t}^{0.4}$ & & & \\
\hline $2 \mathrm{~Hz} * \mathrm{t}^{0.4}$ & -0.01 & .919 & -0.18 to 0.17 \\
$2 \mathrm{~Hz}-\alpha-\mathrm{BGT}^{*} \mathrm{t}^{0.4}$ & -0.28 & .003 & -0.47 to -0.10 \\
\hline Control* $\mathrm{t}^{0.4}$ & -0.30 & .001 & -0.48 to -0.12 \\
$\alpha$-BGT $\mathrm{t}^{0.4}$ & -0.28 & .018 & -0.51 to -0.05 \\
\hline
\end{tabular}

$C I$, Confidence interval; $\alpha$-BGT, $\alpha$-bungarotoxin.

TABLE E2. Mixed-effect ordinal logistic regression analysis of $\alpha$-motor neuron numbers, rates of TUNEL-positive motor neurons, and ratios of microglia area/anterior horn area in relation to grouping and time (compared with sham group)

\begin{tabular}{|c|c|c|c|c|c|c|}
\hline \multirow[b]{2}{*}{ Group (t) } & \multicolumn{2}{|c|}{ Numbers of $\alpha$-motor neuron } & \multicolumn{2}{|c|}{$\begin{array}{l}\log (\text { TUNEL-positive/1- TUNEL- } \\
\text { positive motor neuron rate) }\end{array}$} & \multicolumn{2}{|c|}{$\begin{array}{c}\log (\text { microglia area ratio/ } \\
\text { 1- microglia area ratio) }\end{array}$} \\
\hline & 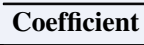 & $95 \% \mathrm{CI}$ & Coefficient & $\mathbf{9 5} \% \mathrm{CI}$ & $\overline{\text { Coefficient }}$ & $\mathbf{9 5} \% \mathrm{CI}$ \\
\hline Sham (Ref) & 37.7 & 36.7 to 40.6 & -1.56 & -1.67 to -1.52 & -3.48 & -3.72 to -3.41 \\
\hline $2 \mathrm{~Hz}$ & -0.7 & -6.2 to 0.3 & 0.21 & 0.09 to 0.50 & 0.38 & 0.10 to 0.82 \\
\hline $2 \mathrm{~Hz}-\alpha-\mathrm{BGT}$ & -9.4 & -13.1 to -8.8 & 0.65 & 0.59 to 0.83 & 0.65 & 0.47 to 1.31 \\
\hline Control & -14.0 & -23.7 to -8.9 & 1.05 & 0.97 to 1.37 & 0.81 & 0.68 to 1.84 \\
\hline$\alpha$-BGT & -17.7 & -22.5 to -15.5 & 1.18 & 1.03 to 1.49 & 1.18 & 0.81 to 1.96 \\
\hline
\end{tabular}

TUNEL, Terminal deoxynucleotidyl transferase-mediated dUTP nick end labeling; $\alpha$-BGT, $\alpha$-bungarotoxin; $C I$, confidence interval. 